KI.EINE TEXTE FÜR THEOLOGISCHE UND PHILOLOGISCHE.

VORLESUNGFN UND IIBUNGEN

HERAUSGEGEBEN VON HANS LIETZMANN

\title{
RES GESTAE DIVI AVGVSTI
}

ITERAUSGEGEREN UND ERKLART

VON

DR. ERNST DIFHI.

A. 1) PROFESSOR IX JKNA

PREIS I,20 MIARK

BON N

A. MARCUS UND E. WEBER'S VERLAG

1908 
Handbuch zum Neuen Testament

il.1 Verbindung mit

H. Gressmann

ᄅ. Kostermann

\author{
$\begin{array}{ll}\text { I. Radermacher } & \text { P. Wendland }\end{array}$ \\ herausgegeben von

\section{Hans Lietzmann} \\ Nene Iieferungen :
}

F. Triebergall

Funfte Iieferung: Band III, Bogen 6-I I. Die Briefe des Apostels Paulns. An die Corinther I. Erklärt von $H$ ans Lietzmann. Lex. 8. M. I.60. Subskr.-Preis M. I.44.

Sechste (Doppel) Iieferung : Band II, Bogen I-10. Die Jvangelien. Markus. Unter Mitwirkung von $\mathrm{Hugo}$ Gressmann, erklärt von Erich Klostermann. Lex. 8. M. 2.85. Subskr.Preis M. 2.56.

Siebente Iieferung: Band V, Bogen 4-8. Praktische Anslegung zn Markus. Von Er. Niebergall. Lex. 8. M. I.40. Subskr.Preis M. I.26.

Achte Irieferung. (Aus Band V.) Praltische Auslegung zu Matthäus und I. Cor. Von F. N iebergall. Unter der Presse. Früher erschienen:

Erste Iieferung. (Band III. Briefe des Apostels Paulus Bogen I-5.) An die Römer. Erklärt von Hans Lietzmann. Subskr.-Preis M. I.35. Einzclpreis M. 1.50.

Zweite Iieferung. (Band V I, Bogen I-3 und V 2 Bogen 1-3.) Allgemeine Finleitung zur praktischen Auslegung und praktische Auslegung zum Römerbrief. Von F. Niebergall. Subskr.-Preis M. I.6o. Einzelpreis M. I.80.

Dritte and vierte Iieferang. Band I 2.) Die hellenistischrömische Kultur in ihren Beziehungen zu Judentum and Christentum. Von P. Wendland. Mit 5 Abbildungen und I 2 Tafeln. Subskr.-Preis M. 4.50. Einzelpreis M. 5.- Gebunden M. 7.-.

Dic in sich abgeschlossenen Lieferungen und Bände des Werkes werden einzeln abgegeben.

Prospekte und Textproben stehen zur Verfügung.

\title{
tbergicht thber das Bammelwerk:
}

Band I: I. Grammatik des neutestamentlichen Griechisch von L. R a d e rmacher. 2. Die hellenistisch-römische Kultur in ihren Beziehungen zu Judentum und Christentum von P. Wendland. 3. Die ur. christlichen Literaturformen von $P$. W endland.

Band II: I. Die Synoptiker unter Mitwirkuug von H. Gressmann, erklärt von E. Klostermann. 2. Johannesevangelium, erklärt von E. Klostermann.

Band III: I. Die vier paulinischen Hauptbriefe erklärt von H. L i etzmann. 2. Die neun übrigen paulinischen. Briefe erklärt von E. Klostermann und H. Lietzmann.

Band IV: I. Die Apostelgeschichte, 2. Katholische Briefe, 3. Hebräerbrief, 4. Apokalypse, erklärt von $E$. $K$ los termann und $H$. Lietzmann.

Band V: Praktische Auslegung des neuen Testaments von F. Niebergall. I. Allgemeine Einleitung und praktische Auslegung zu Band II. 2. Praktische Auslegung za Bard III und IV. 